\title{
Effective treatment with icotinib in lung adenocarcinoma with EGFR and ALK co-alterations and brain metastasis
}

This article was published in the following Dove Press journal:

OncoTargets and Therapy

26 October 2016

Number of times this article has been viewed

\section{Chenyang $\mathrm{Ye}^{1, *}$ \\ Ji Wang ${ }^{2, *}$}

Shu Zheng'

Ying Chai ${ }^{3}$

'Cancer Institute, Key Laboratory of Cancer Prevention and Intervention, National Ministry of Education, Provincial Key Laboratory of Molecular Biology in Medical Sciences, The Second Affiliated Hospital, Zhejiang University School of Medicine, ${ }^{2}$ Department of Surgical Oncology, Sir Run Run Shaw Hospital, College of Medicine, Zhejiang University, ${ }^{3}$ Department of Thoracic Surgery, The Second Affiliated Hospital, Zhejiang University School of Medicine, Hangzhou, People's Republic of China

*These authors contributed equally to this work
Correspondence: Ying Chai Department of Thoracic Surgery, The Second Affiliated Hospital, Zhejiang University School of Medicine, 88 Jiefang Road, Hangzhou 310009, People's

Republic of China

Tel $+8657 \mid 87783367$

Fax +86 571 8778 3318

Email chaiy@I26.com
Abstract: We report a rare case of advanced lung cancer with epidermal growth factor receptor and anaplastic lymphoma kinase co-alterations and brain metastasis, in which icotinib treatment was effective for both the primary lung tumor and the brain metastasis. The patient achieved important clinical remission with a progression-free survival for two years. Our treatment strategy appears to be a promising therapeutic approach for this subgroup of patients.

Keywords: lung cancer, brain metastasis, EGFR, ALK, icotinib

\section{Introduction}

Central nervous system (CNS) metastasis from non-small cell lung cancer (NSCLC) is a devastating complication, resulting in reduced quality of life and shortened survival. Molecular evaluation of primary lung cancer is needed for targeted treatment. We describe a case of advanced lung cancer with concurrent epidermal growth factor receptor (EGFR) mutation and anaplastic lymphoma kinase (ALK) rearrangements, in which both the primary lung lesion and the brain metastasis (BM) showed remarkable responses to icotinib.

\section{Case report}

In June 2014, a 62-year-old Chinese male heavy smoker presented to our hospital with recurrent cough and blood-stained sputum. His previous medical history was unremarkable. Physical examination and laboratory test results showed no significant abnormalities. The chest computed tomographic scan revealed a $3.7 \times 2.8 \mathrm{~cm}$ mass in the left lower lung (LLL) (Figure 1). Mediastinal and hilar lymph nodes were not enlarged. A bronchoscopic biopsy was performed, and histopathologic examination confirmed NSCLC with a moderately differentiated tumor histology favoring adenocarcinoma (Figure 2). Subsequent brain magnetic resonance imaging (MRI) indicated BM (Figure 1). Thus, we made a diagnosis of stage IV lung adenocarcinoma with metastasis to brain according to the 7 th American Joint Committee on Cancer (AJCC)/Union for International Cancer Control (UICC) tumor staging system. In light of the patient's advanced age, a history of heavy smoking for over thirty years, and advanced NSCLC with BM, surgical resection for either primary LLL lesion or BM was not feasible. The patient refused to undergo chemotherapy or radiotherapy. EGFR mutation and ALK translocation status were identified by direct Sanger sequencing, real-time polymerase chain reaction, and fluorescence in situ hybridization (FISH) analysis, and both were 

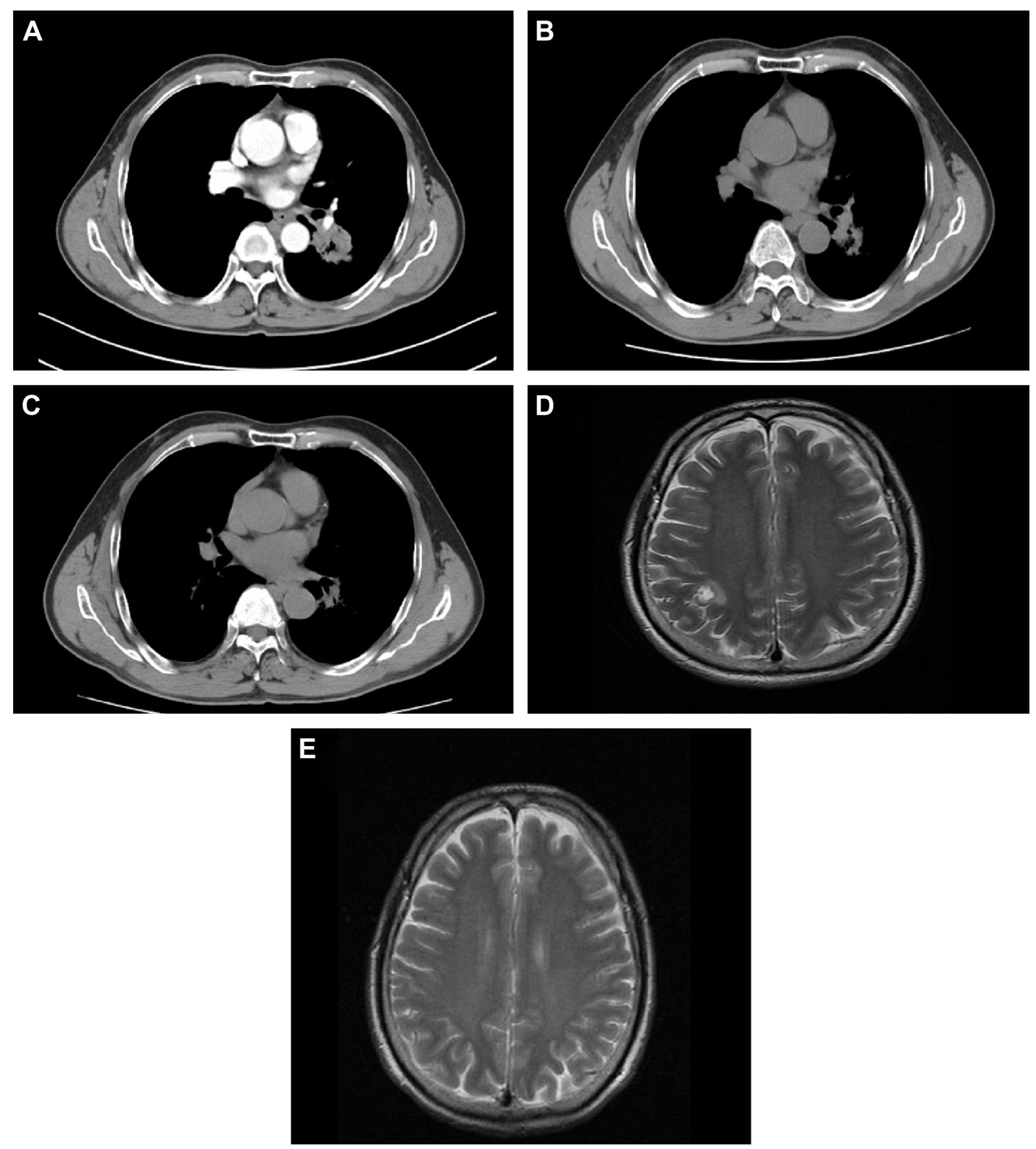

Figure I Management of primary lung cancer and brain metastasis during icotinib treatment. Chest computed tomography (CT) on admission (July 2014) (A) showing a $3.7 \times 2.8 \mathrm{~cm}$ irregularly shaped lesion in the left lower lobe (LLL); the repeated CT one month after icotinib treatment (B) showing a clear reduction in size of the lesion in the LLL; chest CT revealing dramatic shrinkage of LLL mass after one-year maintenance icotinib treatment (C); enhanced brain magnetic resonance imaging (MRI) scan on admission (July 2014) revealing brain metastasis to the right parietal lobe (D); brain MRI obtained in July 2015 showing the intracranial lesion vanished following maintenance icotinib treatment $(\mathbf{E})$.

found to be positive (EGFR exon 21 L858R and ALK FISH positive $22 \%$ of the cells) (Figure $3 \mathrm{~A}$ and $\mathrm{B}$ ). The patient began receiving $375 \mathrm{mg}$ icotinib once daily. A repeated computed tomography (CT) after one month of therapy disclosed that the left lung mass had partially resolved (Figure 1). The patient continued icotinib treatment. In July 2015, a follow-up thoracic CT performed after one year of icotinib therapy revealed dramatic shrinkage of the LLL lesion (Figure 1). Interestingly, brain MRI showed that the intracranial lesion had vanished entirely. He is currently still receiving icotinib treatment, and no recurrence or progression occurred with a progression-free survival for two years. 


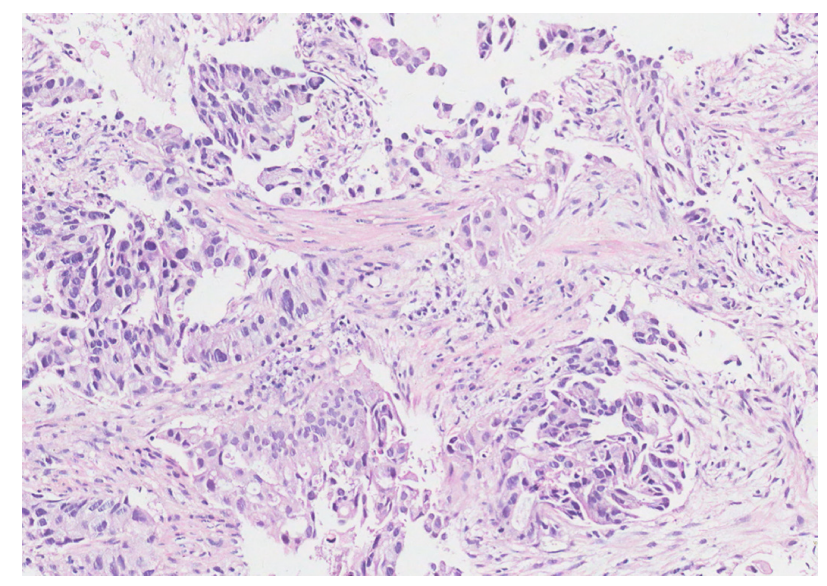

Figure 2 Histology of the primary lung cancer. The hematoxylin and eosin staining revealed that the left lower lobe lesion was a moderately differentiated adenocarcinoma (magnification $\times 100$ ).

\section{Discussion}

The CNS is a common site for NSCLC metastasis, with approximately $30 \%$ to $50 \%{ }^{1}$ of all NSCLC patients ultimately developing BM. Despite recent therapeutic advances, the development of BM from NSCLC remains a significant challenge in treatment. Surgical resection and radiotherapy including whole-brain radiotherapy and stereotactic radiosurgery are currently the most common strategies in the treatment of BM. However, the long-term prognosis of NSCLC patients with BM remains poor, with a median survival of seven months. ${ }^{2}$

Recently, molecular targeted treatments have improved the overall survival of advanced NSCLC patients with specific mutations. ${ }^{3}$ Several studies indicate that molecular targeted therapies, particularly EGFR-tyrosine kinase inhibitors (TKIs) ${ }^{4}$ and crizotinib, ${ }^{5}$ can achieve objective responses of both primary tumors and BM lesions in some patients

A

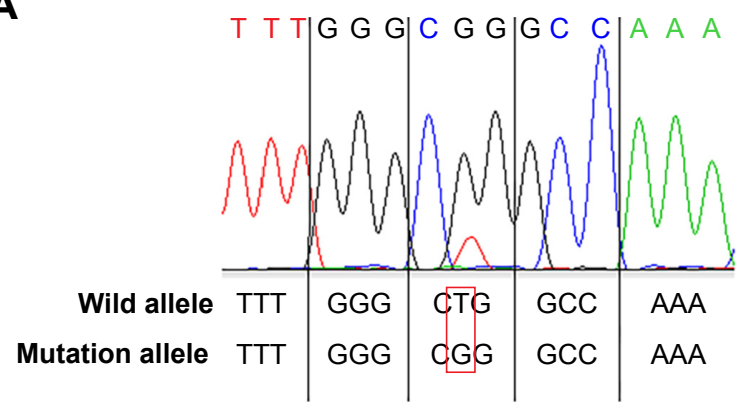

with EGFR-mutated or ALK-rearranged advanced NSCLC. However, effective treatment for NSCLC with EGFR/ALK co-alterations and BM was scarcely reported. EGFR mutations and ALK rearrangements are generally considered mutually exclusive ${ }^{6}$ in NSCLC, and only a few cases have been described in the literature. ${ }^{7,8}$ The best treatment for advanced pulmonary adenocarcinomas with coexisting EGFR mutation and ALK rearrangements remains unclear, because diverse responses to EGFR-TKIs and crizotinib have been previously reported. ${ }^{9}$ Icotinib, an oral EGFR-TKI, has been proved to be non-inferior to gefitinib therapy in patients with NSCLC. ${ }^{10}$ Our patient was prescribed icotinib treatment, and he attained a partial response in the primary left lung lesion and a complete remission in the intracranial metastasis. This rare case obtained important clinical remissions in the primary tumor as well as in the brain lesion for NSCLC patients with dual EGFR/ALK mutations and BM, providing new evidence for targeted therapy in this clinical setting.

\section{Conclusion}

In summary, we present an extremely rare case of advanced lung adenocarcinoma harboring concomitant EGFR mutation and ALK arrangements, in which both the primary lung lesion and the BM responded well to EGFR-TKI treatment. The patient experienced a long-standing response to icotinib with a progression-free survival for two years. This case indicates that molecular evaluation of primary tumor is warranted to test for known driver mutations in the management of advanced NSCLC with BM, providing opportunities for molecular targeted therapy. Our present case provides insight into the efficacy of icotinib for advanced NSCLC patients with co-altered EGFR and ALK. However, further studies to clarify the underlying biological mechanism of EGFR-TKI

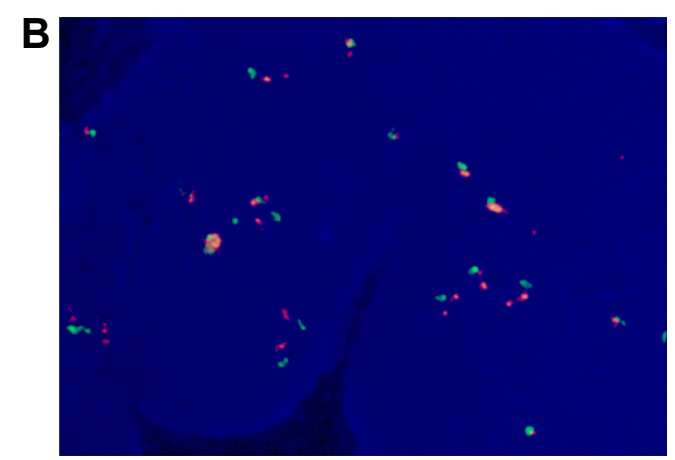

Figure 3 Direct Sanger sequencing and fluorescence in situ hybridization (FISH) analysis of the biopsied lung lesion. A mutant form of EGFR L858R in exon 2I (A) was identified in the left lower lobe (LLL) lesion. Anaplastic lymphoma kinase (ALK) rearrangement was detected by FISH analysis using break-apart probes. Samples are considered to be FISH positive if split ALK $5^{\prime}$ and $3^{\prime}$ probe signals or isolated $3^{\prime}$ signals are detected in at least $15 \%$ of counted tumor cells. Approximately $22 \%$ of lung adenocarcinoma cells indicate ALK rearrangement, as demonstrated by split red/green signals (B).

Abbreviation: EGFR, epidermal growth factor receptor. 
efficacy and the optimal targeted therapy in this patient population are warranted.

\section{Acknowledgments}

The patient provided written informed consent to publish their data and images in this report. The authors thank all the group members for helpful discussions. This work was supported by grants to Y Chai from the Science and Technology Department of Zhejiang Province (2013c03044-7) and the Natural Science Foundation of Zhejiang Province (LY13H160016).

\section{Disclosure}

The authors report no conflicts of interest in this work.

\section{References}

1. Patchell RA. The management of brain metastases. Cancer Treat Rev. 2003;29(6):533-540.

2. Sperduto PW, Chao ST, Sneed PK, et al. Diagnosis-specific prognostic factors, indexes, and treatment outcomes for patients with newly diagnosed brain metastases: a multi-institutional analysis of 4,259 patients. Int J Radiat Oncol Biol Phys. 2010;77(3):655-661.
3. Zhang I, Zaorsky NG, Palmer JD, Mehra R, Lu B. Targeting brain metastases in ALK-rearranged non-small-cell lung cancer. Lancet Oncol. 2015;16(13):e510-e521.

4. Porta R, Sanchez-Torres JM, Paz-Ares L, et al. Brain metastases from lung cancer responding to erlotinib: the importance of EGFR mutation. Eur Respir J. 2011;37(3):624-631.

5. Costa DB, Shaw AT, Ou SH, et al. Clinical experience with crizotinib in patients with advanced ALK-rearranged non-small-cell lung cancer and brain metastases. J Clin Oncol. 2015;33(17):1881-1888.

6. Gainor JF, Varghese AM, Ou SH, et al. ALK rearrangements are mutually exclusive with mutations in EGFR or KRAS: an analysis of 1,683 patients with non-small cell lung cancer. Clin Cancer Res. 2013;19(15): 4273-4281.

7. Kuo YW, Wu SG, Ho CC, Shih JY. Good response to gefitinib in lung adenocarcinoma harboring coexisting EML4-ALK fusion gene and EGFR mutation. J Thorac Oncol. 2010;5(12):2039-2040.

8. Chiari R, Duranti S, Ludovini V, et al. Long-term response to gefitinib and crizotinib in lung adenocarcinoma harboring both epidermal growth factor receptor mutation and EML4-ALK fusion gene. J Clin Oncol. 2014;32(9):e30-e32.

9. Yang JJ, Zhang XC, Su J, et al. Lung cancers with concomitant EGFR mutations and ALK rearrangements: diverse responses to EGFR-TKI and crizotinib in relation to diverse receptors phosphorylation. Clin Cancer Res. 2014;20(5):1383-1392.

10. Shi Y, Zhang L, Liu X, et al. Icotinib versus gefitinib in previously treated advanced non-small-cell lung cancer (ICOGEN): a randomised, double-blind phase 3 non-inferiority trial. Lancet Oncol. 2013; 14(10):953-961.
OncoTargets and Therapy

\section{Publish your work in this journal}

OncoTargets and Therapy is an international, peer-reviewed, open access journal focusing on the pathological basis of all cancers, potential targets for therapy and treatment protocols employed to improve the management of cancer patients. The journal also focuses on the impact of management programs and new therapeutic agents and protocols on

\section{Dovepress}

patient perspectives such as quality of life, adherence and satisfaction. The manuscript management system is completely online and includes a very quick and fair peer-review system, which is all easy to use. Visit http://www.dovepress.com/testimonials.php to read real quotes from published authors. 